CASE SERIES

\title{
Endodontic Failure due to Variations in Root Canal Anatomy in Permanent Mandibular Incisors-A Case Series and Retrospective Pilot Study
}

\author{
Akash More ${ }^{1}$, Sumanthini Margashayam², Vanitha Shenoy ${ }^{3}$, Saima Khan ${ }^{4}$, Gorakh Beble ${ }^{5}$
}

\begin{abstract}
The success of endodontic therapy depends on complete debridement and disinfection of the root canal system. Root canal therapy is based on accessing all areas in a root canal with mechanical and chemical means. Permanent mandibular incisors are generally perceived as teeth with a single root canal but the existence of a second canal has been pointed out. Inability to locate and disinfect the second canal in mandibular incisors leads to persistent periapical inflammation and failure of root canal treatment. Once this canal is located during the endodontic retreatment of these teeth, resolution of symptoms occurs. This case series will highlight the nonsurgical retreatment of permanent mandibular central and lateral incisors where the main cause of failure was a missed canal. In addition, a retrospective pilot study was carried out to find the endodontic failure of mandibular incisors owing to a missed canal. It was also observed that $73 \%$ of teeth had a single canal and $27 \%$ had a complex canal anatomy. A retrospective pilot study was carried out using seventy-five mandibular incisors that were indicated for root canal treatment and were evaluated using digital radiographs. It was also observed that $73 \%$ of teeth had a single canal and $27 \%$ had a complex canal anatomy. This case series will highlight the nonsurgical retreatment of permanent mandibular central and lateral incisors where the main cause of failure was a missed canal. It also throws light on different techniques to diagnose presence of a missed canal if any.
\end{abstract}

Keywords: Endodontics, Incisors, Mandibular, Retreatment.

Journal of Contemporary Dentistry (2019): 10.5005/jp-journals-10031-1247

\section{INTRODUCTION}

One of the reasons for the failure of endodontic therapy is the ignorance of the anatomical variation of the root canal, lack of experience, and skills to negotiate the canal. The dentist must have a thorough knowledge of root canal morphology before starting endodontic treatment. For good prognosis following root canal treatment, the entire root canal system must be explored, adequately debrided, and obturated.

Vertucci et al. used cleared teeth in which the root canal systems were stained with hematoxylin dye and identified eight pulp space configurations which are as follows ${ }^{2}$ (Fig. 1): type I: a single canal extends from the pulp chamber to the apex (1). Type Il: two separate canals leave the pulp chamber and join short of the apex to form one canal (2-1). Type III: one canal leaves the pulp chamber and divides into two in the root; the two then merge to exit as one canal (1-2-1). Type IV: two separate, distinct canals extend from the pulp chamber to the apex (2). Type V: one canal leaves the pulp chamber and divides short of the apex into two separate, distinct canals with separate apical foramina (1-2). Type VI: two separate canals leave the pulp chamber, merge in the body of the root, and redivide short of the apex to exit as two distinct canals (2-1-2). Type VII: one canal leaves the pulp chamber, divides, and then rejoins in the body of the root, and, finally, redivides into two distinct canals short of the apex (1-2-1-2). Type VIII: three canals from the pulp chamber to the apex (3).

Studies on the root canal morphology of extracted mandibular incisors have reported a prevalence of two canals in $12-35 \%$ of the cases. ${ }^{3}$ An in vitro study done by Lalit et al. on north Indian population showed that $63.75 \%$ of the mandibular incisor teeth had a single canal and $36.25 \%$ of roots possessed two canals, while $6.25 \%$ had two separate apical foramina. ${ }^{4}$ There are very few
${ }^{1-5}$ Department of Conservative Dentistry and Endodontics, MGM Dental College and Hospital, Kamothe, Navi Mumbai, Maharashtra, India Corresponding Author: Akash More, Department of Conservative Dentistry and Endodontics, MGM Dental College and Hospital, Kamothe, Navi Mumbai, Maharashtra, India, Phone: +91 8097452710 , e-mail: akmore33@gmail.com

How to cite this article: More A, Margashayam S, et al. Endodontic Failure due to Variations in Root Canal Anatomy in Permanent Mandibular Incisors-A Case Series and Retrospective Pilot Study. J Contemp Dent 2019;9(1):25-31.

Source of support: Nil

Conflict of interest: None

studies in literature which have evaluated the reason for endodontic treatment failure in mandibular teeth. This case series will highlight nonsurgical retreatment of mandibular incisors and endodontic management of missed canal. The purpose of the retrospective pilot study was to determine the cause for failure following root canal treatment in the mandibular incisor owing to missed canals and canal variations seen in Indian population.

\section{Case Report 1}

A 62-year-old male patient reported with the chief complaint of discolored teeth with respect to the lower front teeth region for 5 years. He had undergone root canal treatment 10 years ago followed by a tooth-colored restoration on the lingual aspect of permanent left mandibular central incisor, lateral incisor, and canine without any complications. On clinical examination, tooth colored restoration was noted lingually with 31 and 32 which appeared discolored. Pain on 


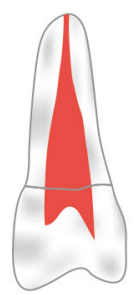

A

Type I

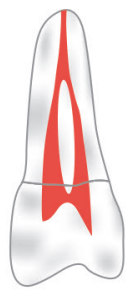

Type II

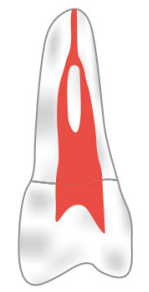

Type III

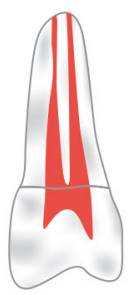

B Type IV

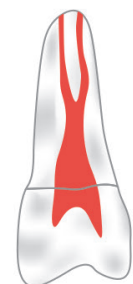

Type $\mathrm{V}$

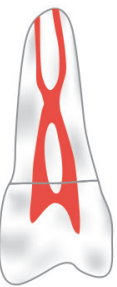

Type VI

Figs $1 \mathrm{~A}$ to $\mathrm{C}$ : Diagrammatic representation of Vertucci's canal configuration ${ }^{2}$

percussion was absent and periodontal probing depth was within normal limits $(<3 \mathrm{~mm})$ with physiological mobility. Pulp vitality testing (Digitest II, USA) gave no response with 31 and 32. Mesial and distal angled radiographic images (Carestream CS 2100, Atlanta, USA) revealed linear radiopacity in coronal and radicular portions suggestive of an obturating material short of the apex with diffuse periapical radiolucency. Radiolucency was seen adjacent to obturating material indicative of a missed root canal on a mesial angulation radiograph (Fig. 2A). Diagnosis of previously root canal-treated teeth with asymptomatic apical periodontitis was arrived at. Patient's medical history was noncontributory. Nonsurgical endodontic retreatment was decided for teeth nos. 31 and 32. Informed consent was obtained from the patient and treatment initiated.

The shape, size, and coronal extent of the tooth-colored restoration were estimated from a diagnostic radiograph. Under rubber dam (Coltene rubber dam kit, Hygienic Switzerland), initial penetration of restoration was made in the middle of the lingual surface of 31 and 32 using a no. 2 round bur (Mani Inc, Japan), just above the cingulum almost perpendicular to the lingual surface using a high-speed contra-angle handpiece. After penetration of the restoration, the bur was directed along the long axis of the tooth until the gutta-percha (GP) was seen. After visualizing GP under dental loupes (Max view, England), long tapered diamond (Mani Inc, Japan) was used to extend the access buccolingually. Coronal GP was removed with gates glidden drill (Mani Inc, Japan) to gain space to deposit xylene (Fischer scientific Thermo Electron LLS India Pvt Ltd) to soften GP which was later removed using the $\mathrm{H}$ file \#15-40 (Mani Inc, Japan). Finally, the paper point wicking technique was carried out where the canal was flushed with xylene followed by introducing paper points \#30 (Diadent, Korea) to absorb the solvent. ${ }^{5}$ Visible traces of softened GP were seen on the paper point. This procedure was repeated until no GP was visible on the paper point. Removal of GP was confirmed radiographically (Fig. 2B). Electronic apex locator (EAL) (J Morita, Japan) was used to determine the tentative working length of the teeth and was confirmed radiographically (Figs $2 \mathrm{C}$ and D). Root canals were then cleaned and shaped using $2 \%$ hand $\mathrm{K}$ and $\mathrm{H}$ files to master apical file (MAF) \#35 using the standardized technique. Master GP cone $2 \%$, \#35 was selected and confirmed radiographically (Mani Inc, Japan)
(Figs $2 \mathrm{E}$ and $\mathrm{F}$ ). A total of $25 \mathrm{~mL}$ of $5 \%$ of sodium hypochlorite (Trifarma Pvt Ltd, Bhiwandi, Thane, India) was used for irrigation using a 27 gauge needle. The smear layer was removed using $17 \%$ of ethylenediaminetetraacetic acid (EDTA Dentwash, Prime Dental Products, Thane, India) solution and was ultrasonically activated using the Irrisafe ultrasonic tip \#25 (Acteon, Merignac, France) at a power setting of 3 with a water outlet kept off. Copious saline irrigation was done before each irrigant change. This was followed by $5 \mathrm{~mL}$ of $2 \%$ of chlorhexidine digluconate (Dentachlor, Ammdent, Mohali, India) that was flushed into the canal and was left undisturbed for 5 minutes followed by copious irrigation with saline. The canals were dried using absorbent points. Calcium hydroxide powder (Prime Dental Products, Thane) was mixed with saline and was placed by indirect ultrasonic activation using \#20 K file kept $1 \mathrm{~mm}$ short of the apex at a power setting of 3 with a water outlet kept off. Obturation was done with GP (Mani Inc, Japan) and AH Plus sealer (Dentsply India Pvt. Ltd) using cold lateral compaction (Figs $2 \mathrm{G}$ and $\mathrm{H}$ ). Vertucci type III canal configuration can be appreciated on an obturation radiograph. The access cavity was temporized with the zinc oxide eugenol (ZOE) cement (Prime Dental Products, Thane, India). Six months follow-up radiograph revealed a resolution of periapical radiolucency with 31 and 32 (Fig. 2l).

\section{Case Report 2}

A 21-year-old male patient reported with the chief complaint of pain with respect to the lower front teeth region for 5 days. He presented with a history of root canal treatment initiated with 32 two months ago. On clinical examination, dislodged restoration was seen lingually with an empty root canal space and grade I mobility with respect to 32 . Pulp vitality testing gave no response and the tooth was tender on percussion. Radiographs revealed radiolucency in the coronal third suggestive of the access opening, empty canal space with diffuse periapical radiolucency (Fig. 3A). Diagnosis of previously initiated root canal treatment with symptomatic apical periodontitis was made. Patients' medical history was noncontributory. Therefore, nonsurgical endodontic retreatment was decided upon. Informed consent was obtained from the patient and treatment initiated. 


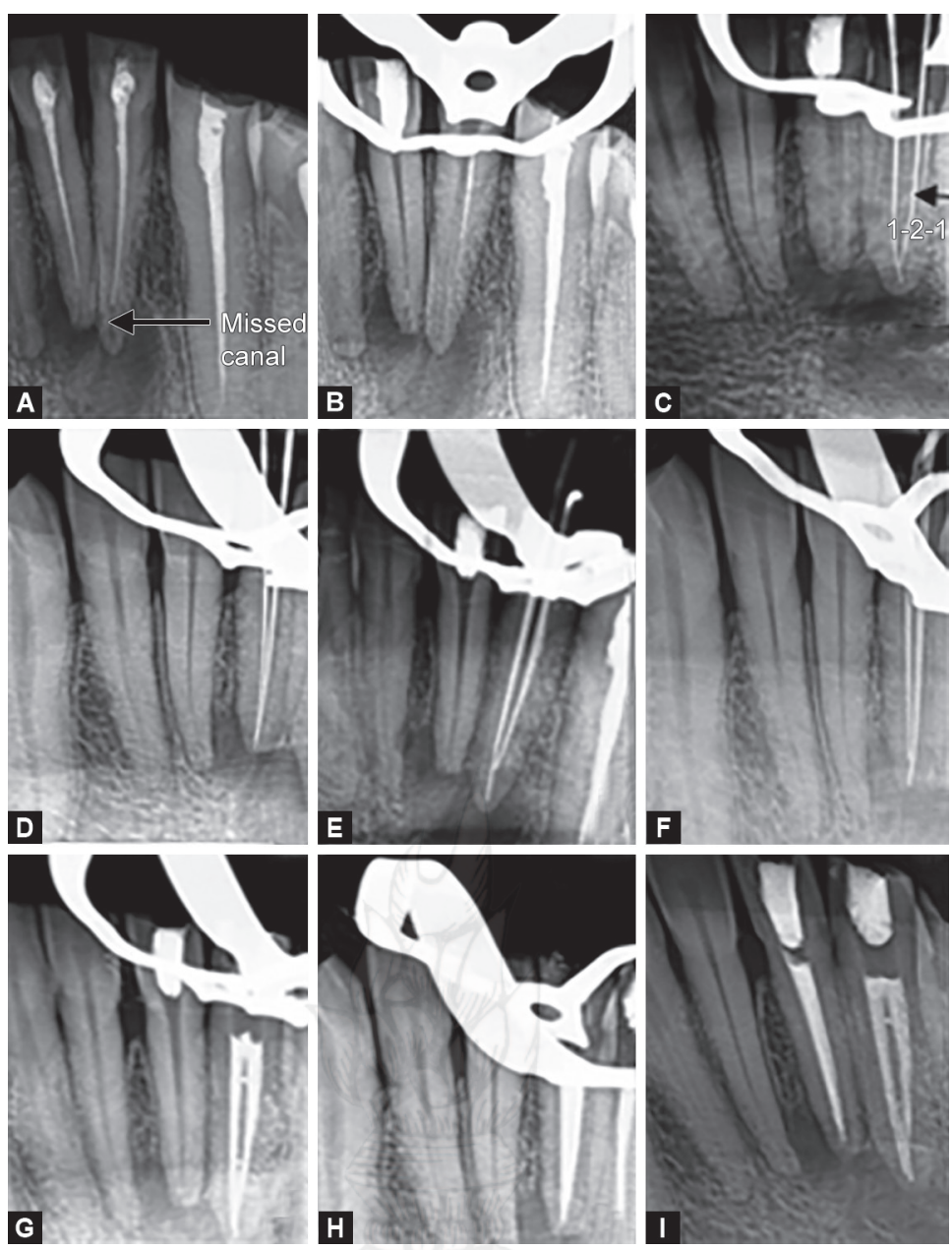

Figs 2A to I: (A)Preoperative intraoral periapical radiograph (IOPA) showing unsatisfactory obturation and diffuse periapical lesion; (B) Radiograph of 31, 32 showing complete removal of root canal filling material; (C) Working length radiograph of 32 showing Vertucci type III canal configuration (1-2-1); (D) Working length radiograph of 31; (E) Master cone selection radiograph of 32; (F) Master cone selection radiograph of 31; (G) Obturation radiograph of 32 showing adequate filling of Certucci type III canal along with filling of isthmus area; (H) Obturation radiograph of 31 ; (I) Six months recall radiograph showing resolution of periapical radiolucency

Under rubber dam isolation, access opening was modified by extending it labiolingually. The un-instrumented lingual canal was scouted using the \#10 K file. The tentative working length was established using an apex locator and was confirmed radiographically (Fig. 3B). Vertucci type $V$ canal configuration was seen on the working length radiograph. A similar technique for cleaning-shaping and intracanal medicament placement was carried out as mentioned in case 1. Master cone was selected and confirmed radiographically (Fig. 3C). Obturation was done using GP and AH Plus sealer using cold lateral compaction followed by the warm vertical compaction technique (Fig. 3D). Vertucci type $\mathrm{V}$ canal configuration can be appreciated on obturation radiograph. The access cavity was temporized with the ZOE cement. Three months follow-up radiograph revealed a resolution of periapical radiolucency with 32 (Fig. 3E).

\section{Case Report 3}

A 25-year-old female patient reported with the chief complaint of pain with respect to the lower front teeth region for 5 days. She had undergone root canal treatment with teeth nos. 32 and 42 six months ago followed by a tooth-colored restoration which dislodged 2 months ago. A metallic object was seen at the canal orifice. Pulp vitality testing gave no response and there was pain on percussion in the concerned teeth. Radiographs revealed linear radiopacity extending beyond apex by $1 \mathrm{~mm}$ with 32 and 42 suggestive of a silver point obturation (Fig. 4A). Radiolucency was seen surrounding the silver point indicating missed canal. A diagnosis of previous root canal treatment with symptomatic apical periodontitis was made with 32 and 42. Patient's medical history was noncontributory. Therefore, nonsurgical endodontic retreatment was decided upon. Informed consent was obtained from the patient and treatment initiated.

Under rubber dam isolation access opening was modified by extending it labiolingually. Dental operating images (DOMs) were recorded (Fig. 5). The access cavity was irrigated with $0.1 \mathrm{~mL}$ of xylene. The solvent was used to soften or dissolve the root canal sealer to enable easy removal. An endodontic explorer and the \#10 file were used to carry the solvent down along the silver point to the dissolve root canal sealer. The Masserann kit (Micro Mega, France) was used for the removal of silverpoint. The coronal length of canal space around the silverpoint was negotiated first using new \#15 H files as far apically as possible. The end-cutting trephine bur was 

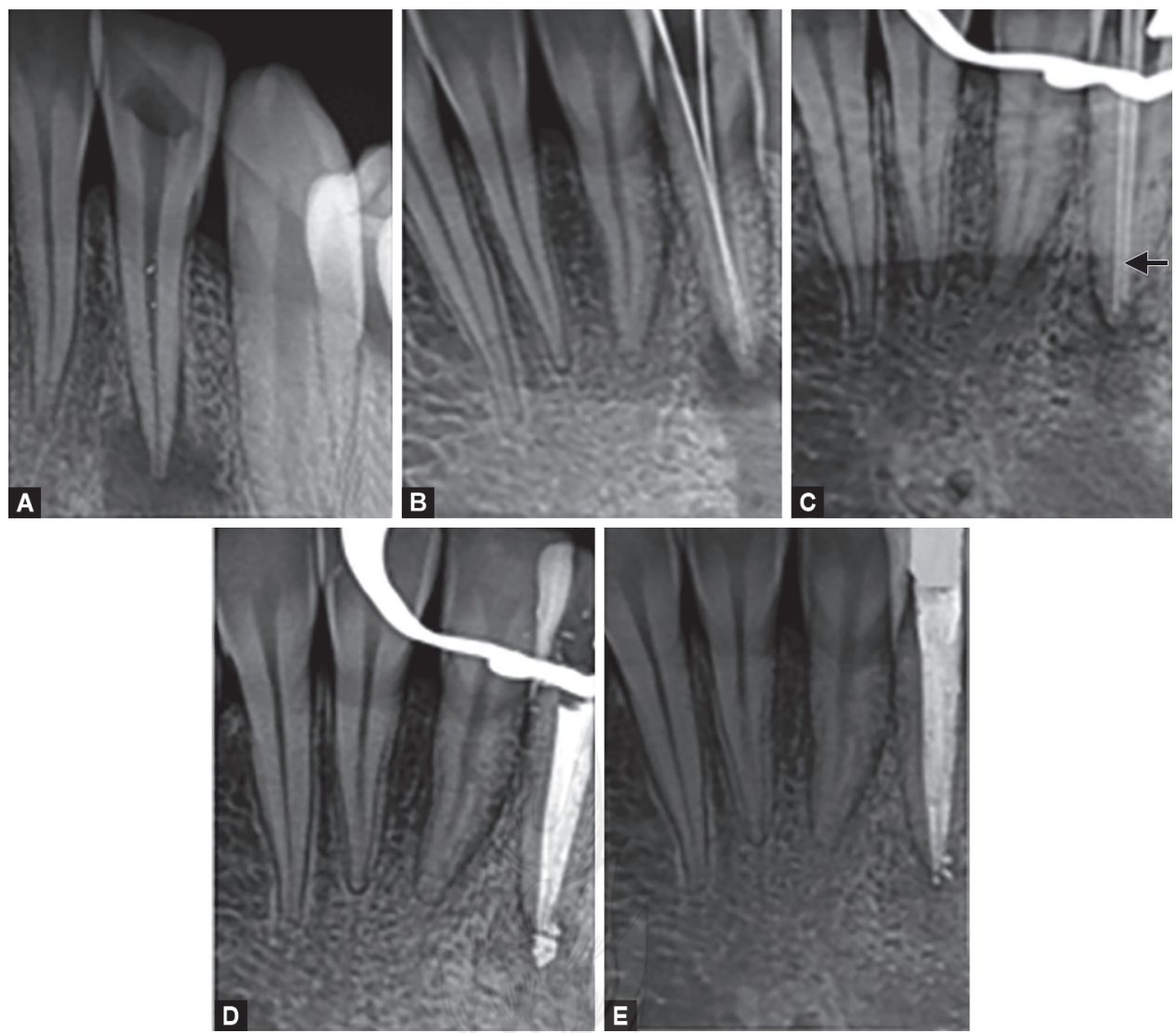

Figs 3A to E: (A) Preoperative IOPA showing unsatisfactory obturation and diffuse periapical lesion with tooth 32; (B) Working length radiograph of 32 shows Vertucci type V canal configuration with a deep split of canal apically; (C) Master cone selection; (D) Obturation radiograph of 32 showing adequate filling of Vertucci type $V$ canal along with sealer extrusion through the two portals of exit at the apex; (E) Three months recall radiograph showing resolution of periapical radiolucency

rotated anticlockwise to create a space around the coronal end of the fragment by cutting the surrounding root canal dentin (Fig. $4 \mathrm{~B})$. The extractor with a plunger rod (stylet) was screwed inside the canal (Fig. 4C). The extractor locks the exposed coronal end of the fragment against internal embossment. It was removed by anticlockwise rotation. The entire length of the silverpoint was removed from 42 and apical one-third of silverpoint was separated for 32 (Fig. 4D). The tentative working length was established using an apex locator and was confirmed radiographically (Fig. 4E). A similar technique for cleaning-shaping and intracanal medicament placement was carried out as mentioned in case 1. A master cone was selected and a radiograph was taken (Fig. 4F). Obturation was done using the GP and the AH Plus sealer using cold lateral compaction and the warm vertical condensation technique (Figs $4 \mathrm{G}$ and H). Vertucci type III canal configuration can be appreciated on obturation radiograph with 32 and 42. The apical separated fragment of silverpoint for 32 was later removed surgically. Six months follow-up radiograph revealed a resolution of periapical radiolucency with 32 and 42 (Fig. 4l).

\section{Retrospective Pilot Study}

Seventy-five mandibular incisors that were indicated for root canal treatment were evaluated using digital radiographs (kodak 5200, USA) at the Department of Conservative Dentistry and Endodontics to study the canal configuration and percentage of endodontic failure of mandibular incisors owing to missed canals in an Indian population. Straight, mesial, and distal radiographs were evaluated to find variations in root canal anatomy according to Vertucci's classification which was later tabulated and evaluated.

\section{Results of the Pilot Study}

The pilot study showed $73 \%$ of cases with the presence of a single canal (type I) and $27 \%$ of cases with the presence of complex canal anatomy among which $7 \%, 13 \%, 6 \%$, and $1 \%$ of cases belonged to types II, III, V, and $\mathrm{VI}$, respectively, as presented in the pie diagram (Fig. 6).

About $87 \%$ of cases reported for conventional root canal treatment owing to caries and trauma, while $13 \%$ of cases reported for Re-RCT as presented in the pie diagram (Fig. 7). Interestingly, all the cases scheduled for retreatment failed due to the presence of a missed canal with unsatisfactory obturation.

\section{Discussion}

The indications for root canal retreatment as stated in a Consensus report of the European Society of Endodontology are the following: ${ }^{6}$

- Teeth with an inadequate root canal filling with radiological findings and/or symptoms.

- Teeth with an inadequate root canal filling when the coronal restoration requires replacement.

GP in combination with a root canal sealer is most often used to obturate the pulp space. Historically, the use of silver points for 


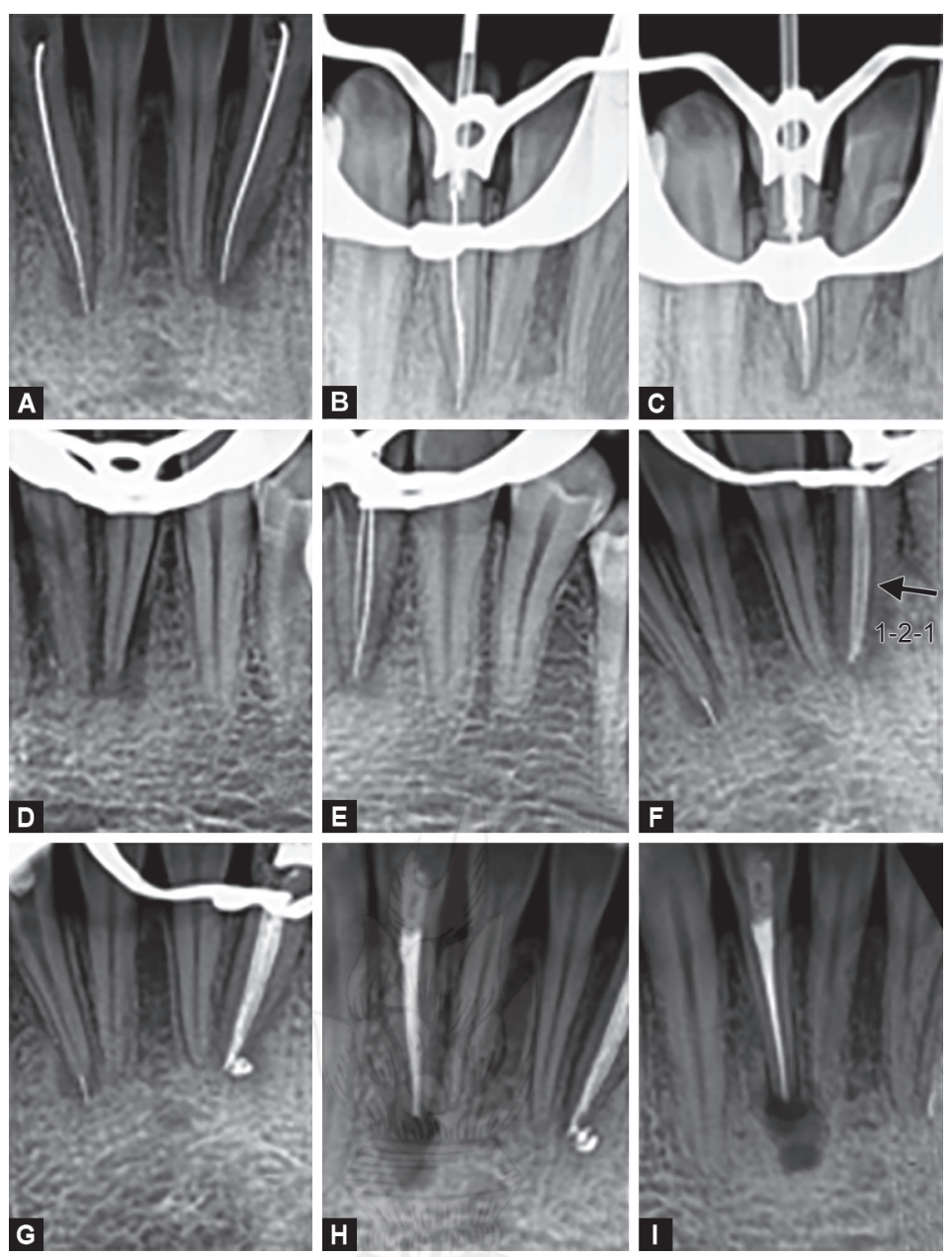

Figs 4A to I: (A)IOPA showing over extended silver point obturation with 32 and 42; (B) IOPA showing trephan bur in position with 32; (C) Extractor in position for 32 silver point removal; (D) IOPA showing removal of silver point; (E) Working length radiograph of 32 shows Vertucci type III canal configuration (1-2-1); (F) Master cone selection radiograph of 32; (G) Obturation radiograph of 32 showing adequate filling of Vertucci type III canal along with sealer extrusion through the portals of exit at the apex; $(\mathrm{H})$ Obturation radiograph of 31 showing adequate filling of Vertucci type III canal configuration; (I) Six months recall radiograph showing resolution of periapical radiolucency

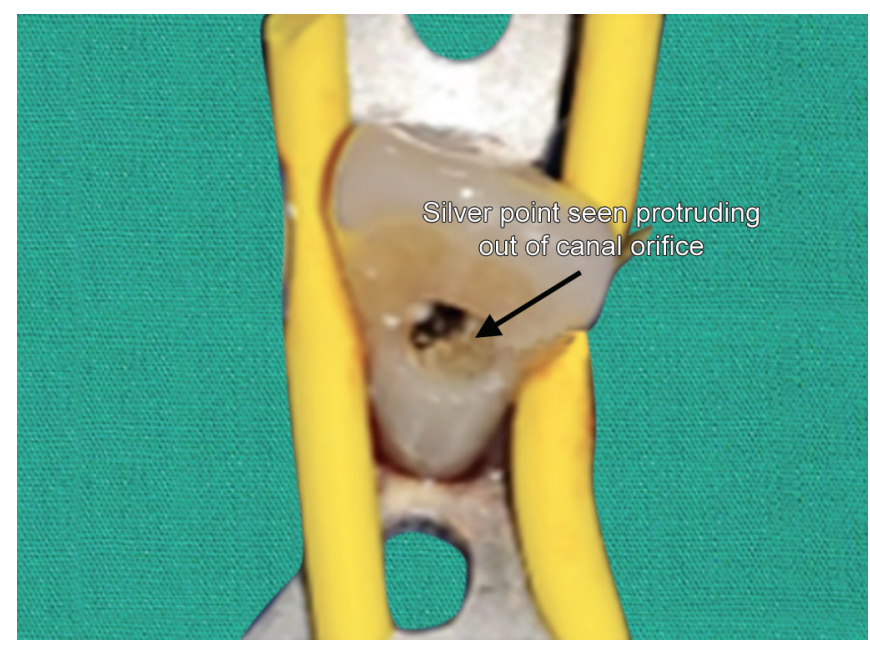

Fig. 5: DOM image showing silver point at the coronal orifice with 32

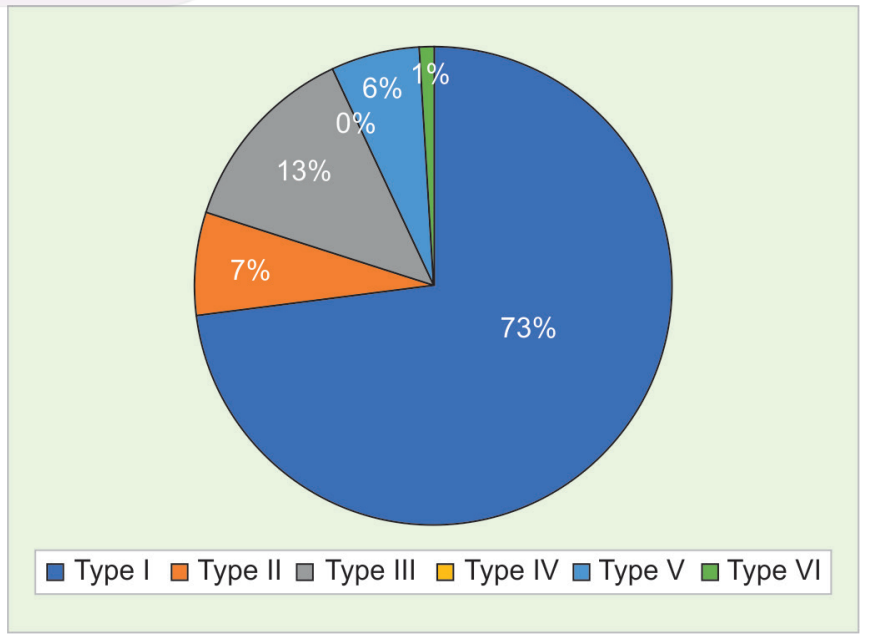

Fig. 6: Retrospective pilot study results showing the distribution of mandibular incisors canal configuration according to Vertucci's classification 


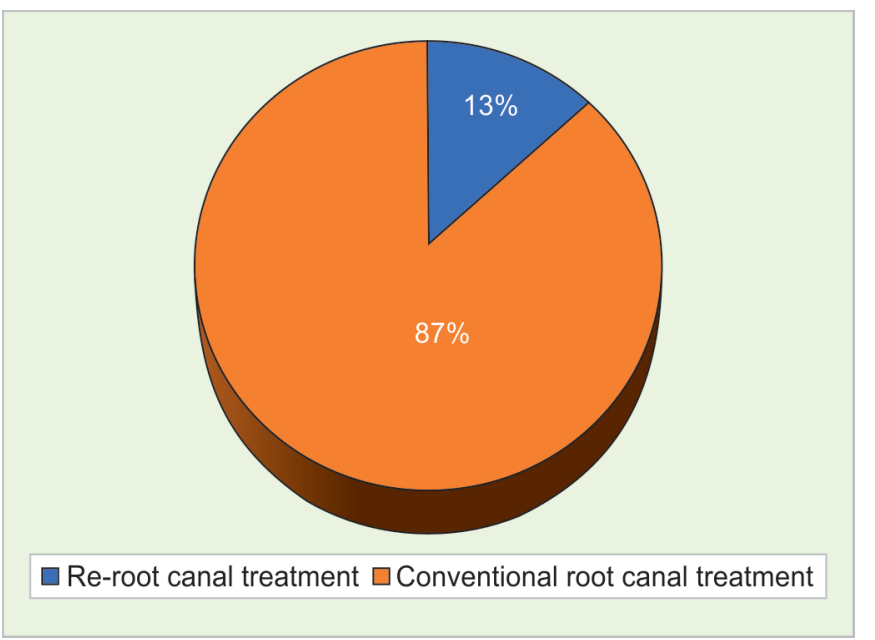

Fig. 7: Percentage of failure following root canal treatment in mandibular incisors

endodontic therapy had been popular because of their ease of handling, placement, ductility radiopacity, and also silver appears to have some antibacterial activity. However, over the past few decades, the usage of silver points has dramatically diminished because they corrode over time and the apical seal is lost. ${ }^{7}$ The corrosion of silver points occurs when they come in contact with tissue fluids and certain chemicals used in endodontics, including sodium hypochlorite and some sealers. ${ }^{8}$ This corrosion produces chemicals such as silver sulfide, silver sulfate, silver carbonate, and silver amine hydrate, which have been shown to be cytotoxic in tissue culture. Corrosion occurs mainly at the apical and coronal portions of the points, indicating that leakage is responsible. Presently, they are considered a deviation from the standard of care. ${ }^{9}$

In the case reports presented, the Vertucci type III canal configuration (1-2-1) (Fig. 1) was seen in cases 1 and 3. The Vertucci type $V$ canal configuration (1-2) (Fig. 1) was seen in case no. 2. The results of the retrospective pilot study showed that $27 \%$ of canals had complex canal anatomy among which the most commonly seen anatomy was the Vertucci type III (1-2-1). Similar results were reported by Lalit et al. where they used canal staining and a tooth-clearing technique on extracted teeth to find the morphological characteristics of the root canal of mandibular incisors in north-east Indian population. ${ }^{4}$

A missed canal, inadequate debridement, and obturation are the main causes for a failed endodontic treatment resulting in a diffuse periapical radiolucency developing over a period of time. ${ }^{10}$ There is a high prevalence of multiple canals in mandibular incisors. Traditional access involves large, triangular, and funnel-shaped coronal preparation to adequately debride the chamber of all pulp remnants. An incisal extension allows better access for instruments and filling materials used in the apical third of the canal. ${ }^{11}$ Access cavity preparation can be modified to facilitate the detection of extra canals and a more incisal approach has to be taken which is more conservative (Fig. 8). ${ }^{12}$

Conservative incisal access is long oval with the greatest width inciso-gingivally and incisal extent closely to the incisal edge. The bur must be angled along the long axis of tooth. As long as the tapered fissured diamond bur remains in the center of tooth parallel to the long axis, the pulp will be reached. To determine true root angulation, the dentist must obtain radiographs and performs the clinical examination before beginning the access. ${ }^{13}$

In patients as an isolated symptom, heat sensitivity may indicate a missed canal. ${ }^{14}$ An anatomic axiom is that if a root contains only a single canal that canal will be positioned close to the center of the root. ${ }^{15}$ If a single canal is located initially on access preparation, an instrument should be placed in the canal and a radiograph must be taken either mesial or distal angulation to find additional canals. If the instrument is skewed considerably off center, the additional canal must be suspected. ${ }^{16}$

All the teeth had undergone endodontic treatment failure. A standardized technique was used for reinstrumentation of the root canals to prevent over the preparation of canals compromising on root dentin thickness. Two cases had a Vertucci type III canal configuration, the point where the two canals join is more constricted than the preparation at the apex. Hence, if both canals are enlarged to the apex, it would result in an hourglass preparation and apical transportation. Filling such a configuration leaves voids in the apical third and invites treatment failure, particularly if microorganisms or their byproducts remain in the canal. ${ }^{17}$ To prevent this, the canal which has a straight-line access to the apex which is considered as the main canal should be prepared till the apex and the second canal till the point of junction. ${ }^{18}$ Obturation of the main canal should be done first while blocking the orifice of the second canal using a paper point/master cone. Once obturation of the main canal is complete, the second canal should be obturated. ${ }^{19}$

The advent of cone beam computerized tomography (CBCT), high-resolution magnification with magnifying loupes, endoscope, and the dental operating microscope has increased the ability to detect canals and may be used to clinically determine the presence of additional canals. The root canal preparation and obturation
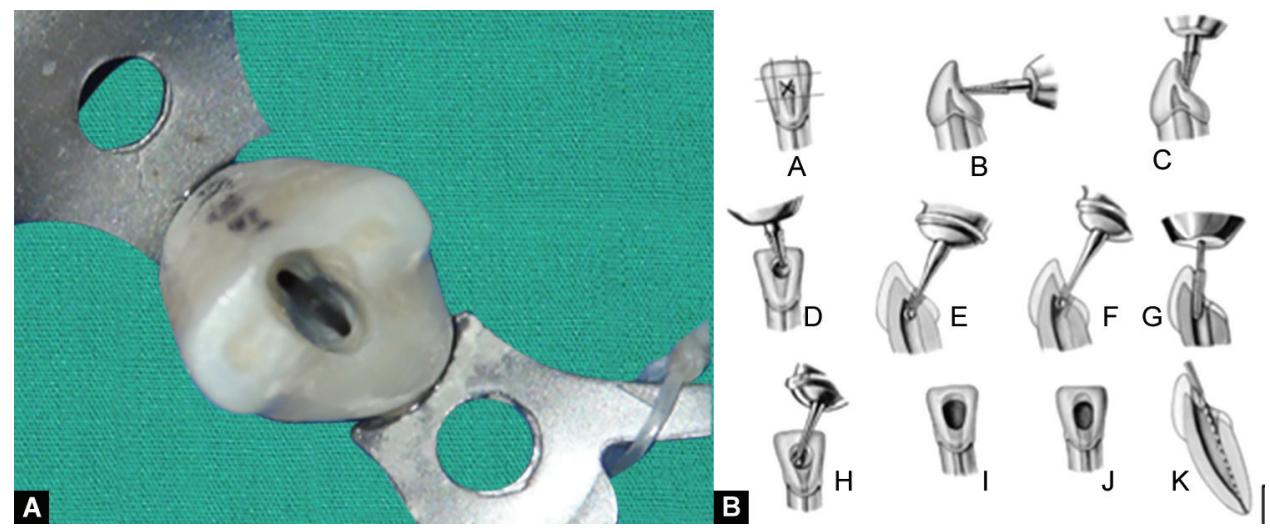

Figs 8A and B: (A) Conservative incisal access cavity preparation in mandibular incisors; (B) Traditional access preparation ${ }^{12}$ 
techniques need to be modified based on the root canal anatomy for successful healing outcomes.

\section{Conclusion}

The possibility of the presence of additional root canals should be kept in mind when treating mandibular anterior teeth. The results of the pilot study show a higher percentage chance of having a second lingual canal in mandibular incisors of the Indian population. Further studies need to be done to determine the canal configuration in Indian population so that endodontic treatment can be carried out predictably.

\section{References}

1. Ingle JI, Bakland LK, et al. Ingle's Endodontics. Endo Cavity Prep, 6th ed. Hamilton: BC Decker Inc, 2008; pp. 560-564; ch. 10.

2. Vertucci FJ. Root canal morphology and its relationship to endodontic procedures. Endod Topics 2005;10(1):3-29. DOI: 10.1111/j.16011546.2005.00129.x.

3. Zhao Y, Dong YT, et al. Cone-beam computed tomography analysis of root canal configuration of 4,674 mandibular anterior teeth. J Pku 2014;46(1):95-99.

4. Lalit C, Bhuyan AC. Morphologic characteristics of root canal of mandibular incisors in North-East Indian population An in vitro study. J Conserv Dent 2011;14(4):346-350. DOI: 10.4103/0972-0707.87195.

5. Ruddle CJ. Nonsurgical endodontic retreatment. Cohen S, Burns RC. Pathways of the pulp, 10th ed. St. Louis: Mosby, 2002; pp. 875-929.

6. Kayaoglu G, Peker I, et al. Root and canal symmetry in the mandibular anterior teeth of patients attending a dental clinic: CBCT study. Braz Oral Res 2015;29(1):1-7. DOI: 10.1590/1807-3107BOR-2015.vol29. 0090.
7. Seltzer S. Endodontology: Biologic considerations in endodontic procedures. Cleaning and shaping, 2nd ed. Philadelphia, 1988; pp. 370-375; ch. 10.

8. Gutierrez JH, Villena F, et al. Microscope and scanning electron microscope examination of silver points corrosion caused by endodontic materials. J Endod 1982;8(1):301-308. DOI: 10.1016/ S0099-2399(82)80279-3.

9. Seltzer S, Green DB, et al. A scanning electron microscope examination of silver cones removed from endodontically treated teeth. Oral Surg Oral Med Oral Pathol 1972;33(2):589-595. DOI: 10.1016/0030-4220(72)90373-8.

10. Ingle Jl, Bakland LK, et al. Ingle's Endodontics. Endo Cavity Prep, 5th ed. Hamilton: BC Decker Inc, 2008; pp. 544-550; ch. 10.

11. Torabinejad M, Walton R. Endodontics: Principles and Practice. Internal Anatomy, 4th ed. St. Louis: Saunders, 2009; pp. 350-355; ch 13.

12. Ingle JI, Bakland LK, et al. Ingle's Endo, 5th ed. Hamilton: BC Decker Inc, 2008; pp. 530-534; ch. 10.

13. Clark D, Khademi J, et al. The new science of strong endo teeth. Dent Today 2013;32(2):112-123.

14. Kartal N, Yanikoglu FC. Root canal morphology of mandibular incisors. J Endodon 1992;18(11):562-568. DOI: 10.1016/S0099-2399(06)81215-X.

15. Cimilli H, Mumcu G, et al. Correlation between root canal patterns. Oral Surg Oral Med Oral Pathol Oral Radiol Endod 2006;102(2):16-20. DOI: 10.1016/j.tripleo.2005.11.015.

16. Pineda F, Kuttler Y. Mesiodistal and buccolingual roentgenographic investigation of 7275 root canals. Oral Surg 1972;33(1):101-107. DOI: 10.1016/0030-4220(72)90214-9.

17. Mauger MJ, Waite RM, et al. Ideal endodontic access in mandibular incisors. J Endod 1999;25(3):206-209. DOI: 10.1016/S0099-2399(99) 80143-5.

18. Hargreaves KM, Cohen S. Cohen's pathways of the pulp. Preparation for Treatment, 10th ed. St Louis: Mosby Inc, 2011; pp. 106-109.

19. Slowey RR. Radiographic aids in the detection of extra root canals. Oral Surg 1974;37(1):762-767. DOI: 10.1016/0030-4220(74)90142-X. 